Draft VERSION July 4, 2018

Preprint typeset using LTEX style emulateapj v. 5/2/11

\title{
GIANT OUTBURSTS IN BE/X-RAY BINARIES
}

\author{
Rebecca G. Martin ${ }^{1,4}$, Chris Nixon ${ }^{1,5}$, Philip J. Armitage ${ }^{1}$, Stephen H. Lubow ${ }^{2}$, and Daniel J. Price ${ }^{3}$ \\ ${ }^{1}$ JILA, University of Colorado \& NIST, UCB 440, Boulder, CO 80309, USA \\ ${ }^{2}$ Space Telescope Science Institute, 3700 San Martin Drive, Baltimore, MD 21218, USA \\ ${ }^{3}$ Monash Centre for Astrophysics (MoCA), School of Mathematical Sciences, Monash University, Vic. 3800, Australia \\ ${ }^{4}$ Sagan Fellow and \\ ${ }^{5}$ Einstein Fellow \\ Draft version July 4, 2018
}

\begin{abstract}
Be/X-ray binary systems exhibit both periodic (Type I) X-ray outbursts and giant (Type II) outbursts, whose origin has remained elusive. We suggest that Type II X-ray outbursts occur when a highly misaligned decretion disk around the $\mathrm{Be}$ star becomes eccentric, allowing the compact object companion to capture a large amount of material at periastron. Using 3D smoothed particle hydrodynamics simulations we model the long term evolution of a representative Be/X-ray binary system. We find that periodic (Type I) X-ray outbursts occur when the neutron star is close to periastron for all disk inclinations. Type II outbursts occur for large misalignment angles and are associated with eccentricity growth that occurs on a timescale of about 10 orbital periods. Mass capture from the eccentric decretion disk results in an accretion disk around the neutron star whose estimated viscous time is long enough to explain the extended duration of Type II outbursts. Previous studies suggested that the outbursts are caused by a warped disk but our results suggest that this is not sufficient, the disk must be both highly misaligned and eccentric to initiate a Type II accretion event.
\end{abstract}

Subject headings: accretion, accretion disks binaries: general stars: emission-line, Be

\section{INTRODUCTION}

Be stars are early main-sequence stars which have shown $\mathrm{H} \alpha$ emission at least once (e.g. Porter \& Rivinius 2003). They are rapidly rotating close to their break up velocity (Slettebak 1982; Porter 1996). The stars are surrounded by decretion disks (Pringle 1991), formed from ejected material that settles into a Keplerian disk and spreads outwards through viscous diffusion (Lee et al. 1991; Hanuschik 1996; Carciofi 2011). The mechanism for ejection is uncertain (rotation alone is insufficient), with magnetic fields (e.g. Underhill 1987; Smith et al. 1994) or stellar pulsation (Rivinius et al. 2001) being candidates. The disk and its associated emission appears and disappears on a timescale of a few months to a few years (e.g. Biorkman et al. 2002).

Gas from the decretion disk of the Be star can be captured and accreted by a companion in Be/X-ray binaries. These systems contain a Be star in a relatively wide orbit (orbital period of tens to hundreds of days) of significant eccentricity $(e \gtrsim 0.1)$ with a compact object, often a neutron star (Reig 2011) or less commonly a black hole (Munar-Adrover et al. 2014). Typically the orbital angular momentum of the binary is misaligned to the spin of the Be star, with a relative inclination of $i \gtrsim 25^{\circ}$ (see Table 1 in Martin et al. 2011 and references therein). Both the misalignment and the orbital eccentricity are plausibly the result of kicks imparted during the supernova that formed the compact object (e.g. Martin et al. 2009, 2010).

In this paper, we investigate how the interaction of the misaligned disk and eccentric binary may give rise to the observed X-ray phenomenology of Be/X-ray binaries. Two types of X-ray outbursts are produced in such systems (Stella et al. 1986; Negueruela et al. 1998). Type I X-ray outbursts are periodic and coincide with periastron passage with a typical luminosity of $L_{X}=10^{36}-10^{37} \mathrm{erg} \mathrm{s}^{-1}$ that lasts for a few days. Type II X-ray outbursts occur much less frequently but have a larger luminosity of $L_{X}>10^{37} \mathrm{erg} \mathrm{s}^{-1}$ and last weeks to months, showing no orbital modulation. The majority of giant outbursts are finished in less than an orbital period, but some may last up to five orbital periods (Kretschmar et al. 2013). Several suggestions as to the origin of these outbursts have been made (Negueruela et al. 2001; Okazaki \& Negueruela 2001; Moritani et al. 2013; Okazaki et al.2013), all of which involve as a central element the tilted and warped disks that have been observed before and during the outbursts (Negueruela et al. 2001; Reig et al. 2007; Moritani et al. 2011). Most recently, Kato (2014), presented analytic arguments suggesting that simultaneous growth of eccentric and tilt modes could occur on time-scales relevant to the giant outbursts. Exactly how a warped (and potentially eccentric) disk causes a long duration accretion event onto the neutron star remains, however, unclear.

The outline of the paper is as follows. In $\$ 2$ we present results from three dimensional numerical simulations of accretion in Be/X-ray binaries. Type I outbursts can be produced when the neutron star captures a small fraction of low angular momentum gas from the disk at closest approach. We show that Type II outbursts can be explained as a consequence of eccentricity growth within the Be star disc. Capture of larger amounts of gas from the eccentric disk generates a Type II outburst, and in $\S 3$ we show that the long duration of these events can be due to the increased viscous time when the material circularizes around the compact object. We discuss our results in $\S 4$ and $\S 5$.

\section{DECRETION DISK AROUND THE BE STAR}

Previous work on misaligned Be star systems mainly focused on coplanar systems or those with a small misalignment angle, $i \lesssim 45^{\circ}$ (e.g. Okazaki 2006; Okazaki \& Hayasaki 2007; Okazaki 2007). They found that the resonant truncation radius for the Be star disk is the same for the inclined case unless $i>60^{\circ}$ ( $i$ is the angle between the spin of the Be star and the binary orbital axis). In this work we use the smoothed particle hydrodynamics (SPH) code . Рнаnтom (Price \& Federrath 


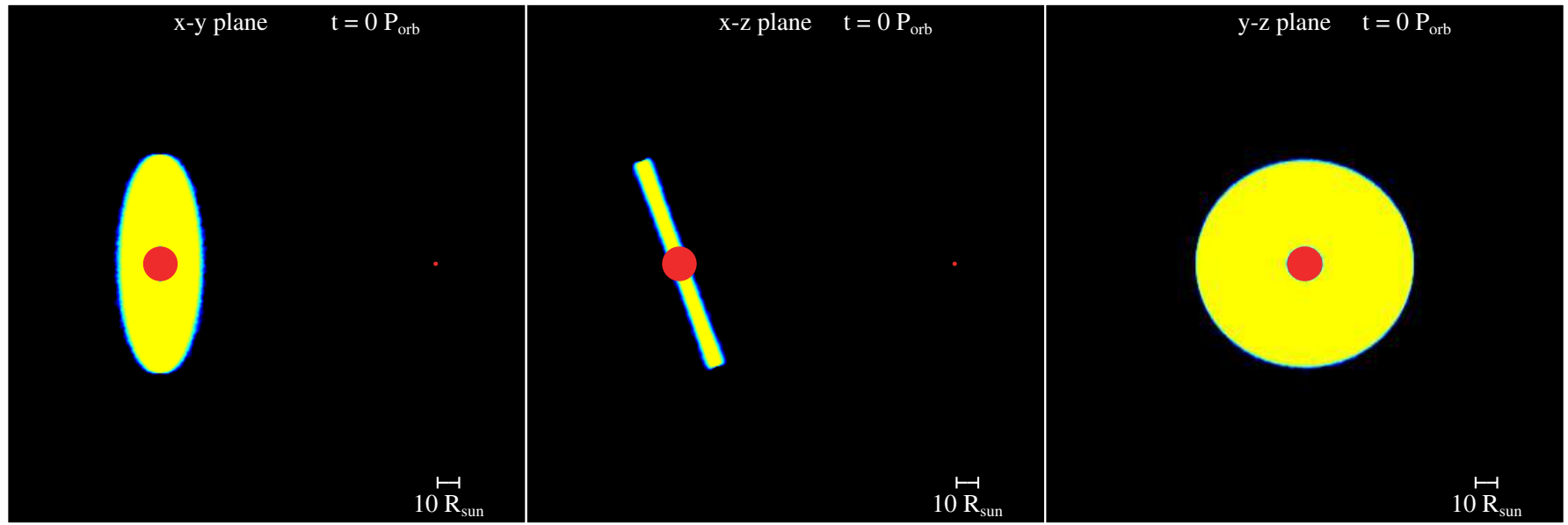

FIG. 1. - Initial conditions for the SPH simulation of a Be star (large red circle) with a disk and a neutron star (small red circle) binary companion. The size of the circles denotes their respective accretion radii. The colour of the gas denotes the column density with yellow being about two orders of magnitude larger than blue. The left panel shows the view looking down on to the $x-y$ binary orbital plane and the middle and right panels show the views in the binary orbital plane, the $x-z$ and $y-z$ planes, respectively. Initially the disk is circular and flat, but tilted from the binary orbital plane by $70^{\circ}$. Note that in the right hand panel the neutron star and the Be star coincide with each other.

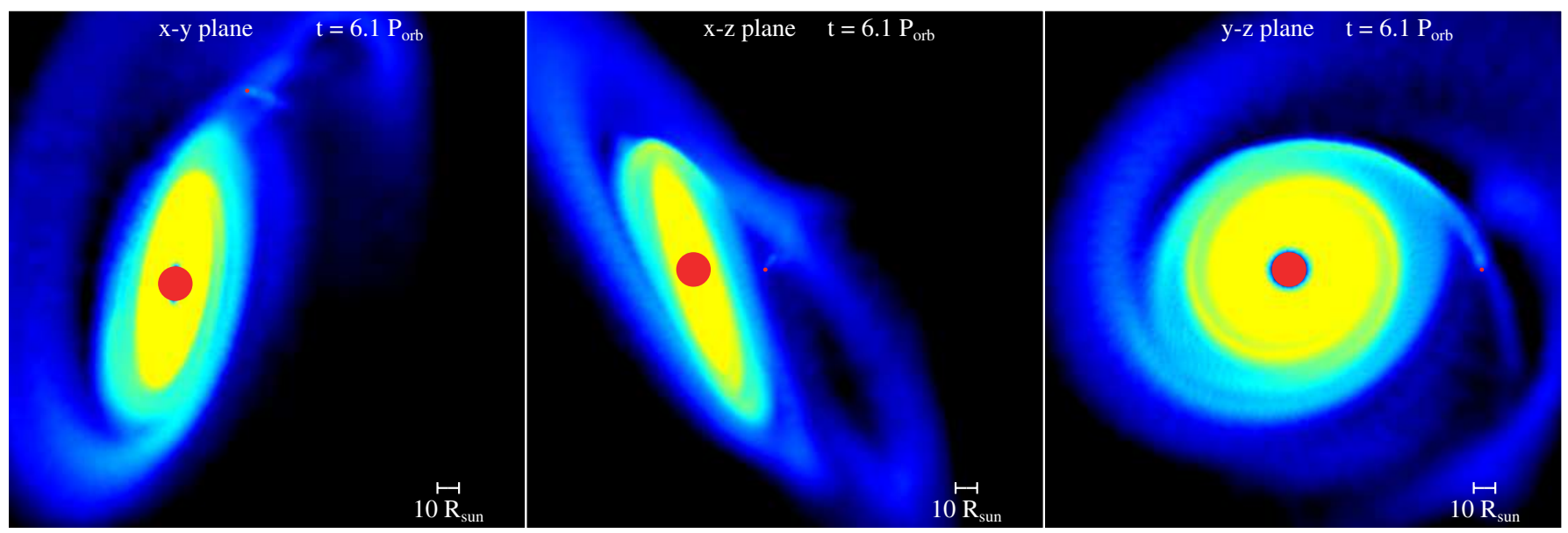

Fig. 2.- Same as Fig. 1 but at a time of $6.1 P_{\text {.orb }}$. The Be star disk is close to circular and the neutron star is moving towards periastron and undergoing a Type I outburst.

TABLE 1

BE/X-RAY BINARY MODEL PROPERTIES

\begin{tabular}{lll}
\hline Be/X-ray Binary Parameters & & \\
\hline \hline Mass of the Be star & $M_{\star} / . \mathrm{M}_{\odot}$ & 18 \\
Radius of the Be star & $R_{\star} / . \mathrm{R}_{\odot}$ & 8 \\
Mass of the neutron star & $M_{\text {.NS }} / . \mathrm{M}_{\odot}$ & 1.4 \\
Mass of the binary & $M / . \mathrm{M}_{\odot}$ & 19.4 \\
\hline Orbital Period & $P_{\text {.orb }} / . \mathrm{d}$ & 24 \\
Semi-major axis & $a / . \mathrm{R}_{\odot}$ & 95 \\
Eccentricity & $e$ & 0.34 \\
\hline Initial Be star disk mass & $M_{\text {.di }} / \mathrm{M}_{\odot}$ & $10^{-8}$ \\
Be star disk viscosity parameter & $\alpha$ & $0.2-0.4$ \\
Be star disk aspect ratio & $H / R\left(R=R_{\star}\right)$ & 0.01 \\
Initial disk inclination & $i$ & $70^{\circ}$ \\
\hline
\end{tabular}

2010; Lodato \& Price 2010) to model highly misaligned systems. Phantom has been used to model misaligned disks in other binary systems (Nixon 2012; Nixon et al. 2013) and we use the same method here. The simulations have two sink particles, one representing a Be star with mass $M_{\star}=18 \mathrm{M}_{\odot}$ and the other a neutron star of mass $M_{\mathrm{NS}}=1.4 \mathrm{M}_{\odot}$. The binary has an orbital period of $P_{\mathrm{orb}}=24 \mathrm{~d}$ (thus the orbit has semimajor axis $a=95 \mathrm{R}_{\odot}$ ) and eccentricity $e=0.34$, to target the
Be/X-ray binary 4U 0115+63, although our results are more generally applicable. The parameters of the simulation are summarized in Table 1. Figs. 1, 3 show the overall evolution of the calculation.

The accretion radius of the Be star is equal to its stellar radius of $R_{\star}=8 \mathrm{R}_{\odot}$. For the neutron star we take the accretion radius to be $1 \mathrm{R}_{\odot}$ (the Bondi-Hoyle accretion radius at periastron is about $3.3 \mathrm{R}_{\odot}$ ). Thus, we do not model the inner parts of the accretion disk around the neutron star (if it forms). Fig. 11 shows the initial flat, but tilted disk with an inclination of $i=70^{\circ}$ to the $x$-axis. The disk has a mass of $M_{\mathrm{di}}=10^{-8} \mathrm{M}_{\odot}$ distributed with a surface density power law $\Sigma \propto R^{-1}$ between $R_{\text {in }}=R_{\star}$ and $R_{\text {out }}=50 \mathrm{R}_{\odot}$ with $10^{6} \mathrm{SPH}$ particles. Varying the outer disk radius, we find little difference in the disk behaviour for smaller disk radii once the disk has expanded to the tidal truncation radius. The disk is isothermal with an aspect ratio $H / R=0.01$ at the inner edge, where $R=R_{\star}$, and the Shakura \& Sunvaev (1973) $\alpha$ parameter varies in the range $0.2-0.4$ (e.g. King et al. 2007) over the disk (we implement the disk viscosity in the usual manner by adapting the SPH artificial viscosity according to the procedure described in Lodato \& Price (2010), using $\alpha_{\mathrm{AV}}=6.0$ and $\beta_{\mathrm{AV}}=2.0$ ). The Be star disc is resolved with shell-averaged smoothing 


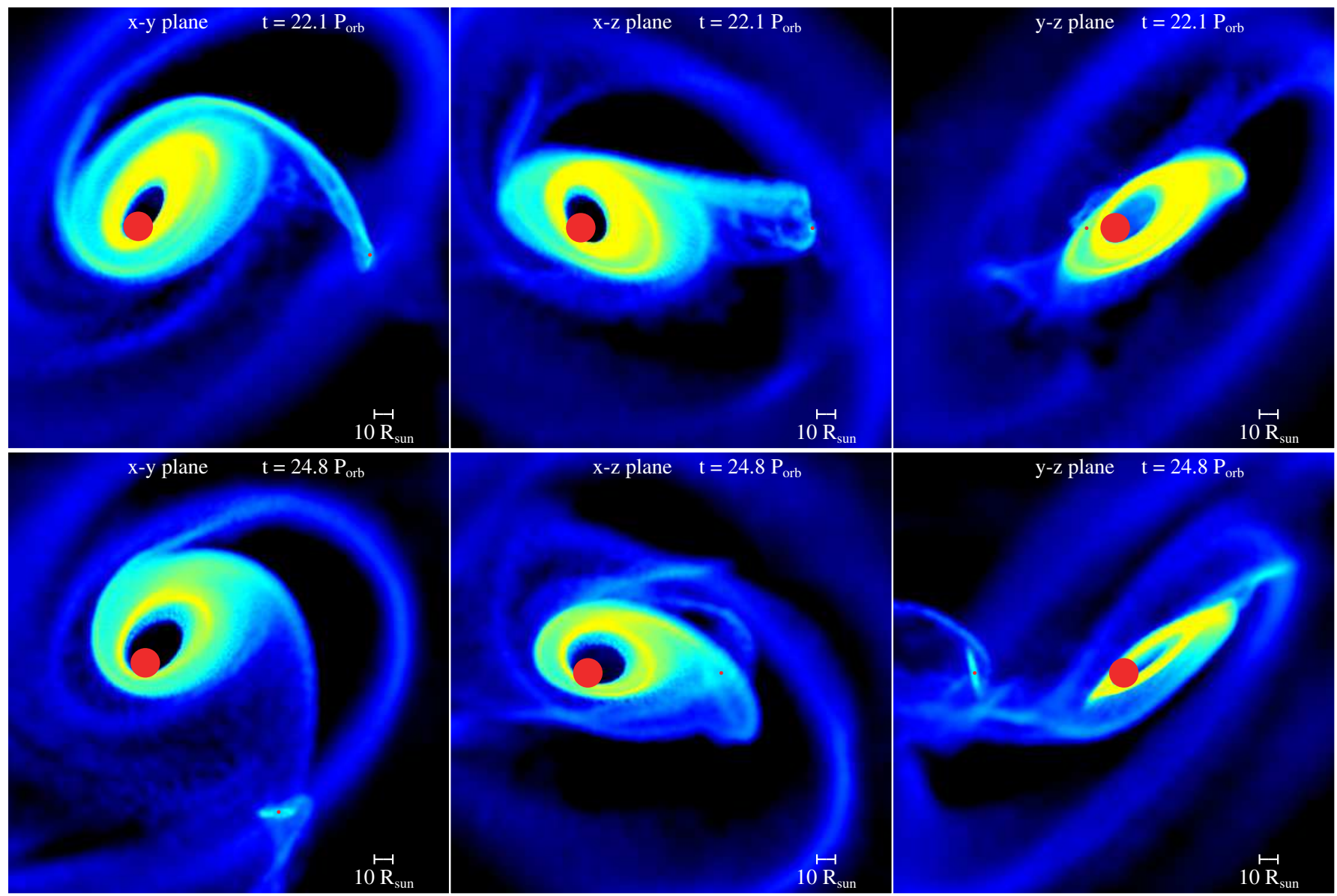

FIg. 3. - Same as Fig. 1 but at times $t=22.1 P_{\text {.orb }}$ (upper panels) and $t=24.8 P_{\text {.orb }}$ (lower panels) during and after the Type II outburst. The Be star disk is eccentric. Top panels: The neutron star is passing through a spiral arm and accreting a high amount of mass in a Type II outburst. Bottom panels: After the Type II outburst a disk has formed around the neutron star.

length per scale height $\langle h\rangle / H=0.5$.

The left hand panel of Fig. 4 shows the mass flow rate through the neutron star accretion radius as a function of time. Initially small (Type I) accretion outbursts occur every orbital period. Fig. 2 2 show the disk structure during a Type I outburst at a time of $6.1 P_{\text {orb. }}$. These outbursts occur when the disk is close to circular and the neutron star passes close to the spiral arm of the Be star disk. A small amount of material is pulled from the spiral arm on to the neutron star.

Fig 4 shows that at a time of around $21 P_{\text {orb }}$ the accretion changes phase. The peak accretion rate is about an order of magnitude higher than during the Type I phase and the accretion does not subside between the periastron passages. We suggest that this is a giant Type II outburst. This can be seen clearly in the right panel of Fig. 4 where we show the total mass accreted on to the neutron star as a function of time. The mass increases slowly during the Type I phase and then dramatically during the Type II phase before returning to the Type I phase. The top row of panels in Fig. 3 show the disk structure during the Type II outburst at a time of $22.1 P_{\text {orb }}$. The Be star disk is highly eccentric and it is this that causes the Type II outburst where a large amount of material is transferred to the neutron star. The bottom row of panels show the end of the outburst and the neutron star disk. The precession of the eccentric Be star disk can also be seen by comparing the disk structures at different times.

We bin the particles in (instantaneous) radius from the $\mathrm{Be}$ star and calculate properties of the Be star disk. Initially the disk is misaligned by $i=70^{\circ}$. Just before the Type II outburst, the disk has aligned to $i \approx 60^{\circ}$. The disk remains approximately flat during this process with the outer parts aligning only a few degrees more than the inner parts. This is in agreement with the analytic estimates for the alignment timescale for the Be star disk with the binary orbit of the order of a few years (Martin et al. 2011). Furthermore, we find that the eccentricity grows first at large radius in the disk. Just before the Type II outburst, the eccentricity of the disk is approximately constant with radius at $e=0.6$. During and after the giant outburst, the eccentricity decays.

\subsection{Eccentricity Growth in the Be star disk}

There are several mechanisms that may drive eccentricity in a circumprimary disk that we discuss here. First, eccentricity may be directly excited due to the eccentric companion. A perturber on an eccentric orbit forces an eccentricity on an initially circular particle orbit. The particle eccentricity grows nearly linearly in time (see Section III.c of Goldreich \& Tremaine (1980)). We have performed test particle simulations of a coplanar eccentric binary system including the eccentric forcing of the companion (see equation (9) in Goldreich \& Tremaine 1980). The initially constant rate of eccentricity growth of the particle increases strongly with distance from the Be star, $d$, as $d e / d t \propto d^{2.8}$ (for $d \lesssim 0.55 a$ ). For example, a particle with an initial distance of $d=0.5 \mathrm{a}$, 
the eccentricity grows linearly at a rate of $d e / d t \approx 0.05$ per binary orbit. Thus, the disk becomes eccentric on a timescale of around 10 orbital periods. The eccentricity of the particle orbit grows perpendicularly to the eccentricity of the binary orbit. This is observed in our simulations and thus we suggest that gravitational forcing from the companion is the most likely mechanism for eccentricity growth here. Hence, the disk must expand radially in order for eccentricity to be driven. We will investigate this process in the inclined disk in more detail in a future publication.

Secondly, instability at the 3:1 Lindblad resonance can lead to eccentricity growth even in an initially circular disk (Lubow $1991 \mathrm{a}, \mathrm{b}, 1992)$. The radius of the $3: 1$ resonance is $R_{\text {res }}=$ $3^{-2 / 3}(1+q)^{-1 / 3} a$ (e.g. Goodchild \& Ogilvie 2006), which for our typical parameters is $R_{\text {res }} \approx 44.5 \mathrm{R}_{\odot}$. Finally, it could be possible for eccentricity to be driven by the stream from the outside of the Be star disk falling back towards and hitting the Be star disk (Lubow 1994; Shi et al. 2012). However, there is only a small amount of material involved in the streams.

In the coplanar case, the tidal truncation radius of the disk may be estimated by the radius at which ballistic particle orbits cross. In a circular binary with our parameters this would be at a radius of $0.48 a$ (Paczynski 1977). For our parameters this is $45.6\left(a / 95 \mathrm{R}_{\odot}\right) \mathrm{R}_{\odot}$. In an eccentric binary, the size of the disk is further reduced. For our parameters, the truncation radius is around $0.38 a$ (Artymowicz \& Lubow 1994). In an eccentric binary, the disk eccentricity (with no dissipation) grows at all radii but the rate is strongly dependent on radius. Thus large eccentricity growth is limited to high inclination disks where reduced tidal torques allow the disk to expand to large radii (Nixon et al. in prep). For smaller disks (lower inclinations), dissipation can keep the disk close to circular with $e \lesssim 0.1$ (Okazaki et al. 2002). We have run several SPH simulations with varying misalignment angle and we find that the disk becomes significantly eccentric only for $i \gtrsim 60^{\circ}$. Thus, Type II outbursts are expected only in systems with a large misalignment angle.

\section{ACCRETION DISK AROUND THE NEUTRON STAR}

Here we consider properties of the accretion disk that forms around the neutron star. Due to the misalignment between the Be star disk and the neutron star orbit it is likely that accretion on to the neutron star proceeds through an initially misaligned disk (c.f. Nixon \& Salvesen 2014). For numerical tractability we cannot model the neutron star disk in its entirety and it remains under-resolved even during the peak of the Type II outburst. This will be investigated in more detail in a future publication. However, we have run several SPH simulations with a much smaller neutron star accretion radius. We do not find significant evidence for a neutron star accretion disk during the Type I activity, only during the Type II. There is much less material and its circularization radius is much smaller during the Type I outburst phase and thus it accretes more rapidly (see also Okazaki et al. 2002).

The viscous timescale in the neutron star disk is $\tau_{v_{\mathrm{NS}}}=$ $R_{\mathrm{NS}}^{2} / \nu_{\mathrm{NS}}$, where $R_{\mathrm{NS}}$ is the distance from the neutron star, the viscosity is $v_{\mathrm{NS}}=\alpha_{\mathrm{NS}} c_{\mathrm{s}, \mathrm{NS}}^{2} / \Omega_{\mathrm{NS}}, \alpha_{\mathrm{NS}}$ is the Shakura \& Sunyaev (1973) parameter and the sound speed is $c_{\mathrm{s}, \mathrm{NS}}=\sqrt{\mathcal{R} T / \mu}$ where $\mathcal{R}$ is the gas constant and $\mu$ is the gas mean molecular weight. The temperature of the disk surface is found by assuming a steady profile

$$
\sigma T^{4}=\frac{3 \dot{M}_{\mathrm{NS}}}{8 \pi} \Omega_{\mathrm{NS}}^{2}
$$

(Pringle 1981). Thus, the accretion timescale of the disk scaled by the orbital period is

$\frac{\tau_{\nu_{\mathrm{NS}}}}{P_{\text {orb }}}=4.7\left(\frac{\alpha_{\mathrm{NS}}}{0.3}\right)^{-1}\left(\frac{M_{\mathrm{NS}}}{1.4 \mathrm{M}_{\odot}}\right)^{\frac{1}{4}}\left(\frac{\dot{M}_{\mathrm{NS}}}{10^{-8} \mathrm{M}_{\odot} \mathrm{yr}^{-1}}\right)^{-\frac{1}{4}}\left(\frac{R_{\mathrm{NS}}}{0.5 \mathrm{R}_{\odot}}\right)_{(2)}^{\frac{3}{4}}$.

If the disc is significantly optically thick this is an overestimate. While a radially extended neutron star disk is present, the accretion on to the neutron star is smoothed on a timescale of several orbital periods. This explains why there is no orbital modulation observed during a Type II X-ray outburst. We do not expect smoothing on this timescale to take place during the Type I activity because the accreting material has lower initial angular momentum and therefore a smaller circularisation radius $\left(\lesssim 0.5 R_{\odot}\right)$ and viscous time. These numbers are approximate and tend to over-predict the viscous time compared to observations as we assume a steady disk. Time dependent processes, such as tides or interactions of the neutron star disk with streams from the Be star disk could shorten the viscous timescale.

The corresponding X-ray luminosity of the disk is $L_{\mathrm{X}}=$ $G M_{\mathrm{NS}} \dot{M}_{\mathrm{NS}} / r_{\mathrm{NS}}$, where $r_{\mathrm{NS}}$ is the radius of the neutron star and thus

$L_{\mathrm{X}}=1.2 \times 10^{38}\left(\frac{M_{\mathrm{NS}}}{1.4 \mathrm{M}_{\odot}}\right)\left(\frac{\dot{M}_{\mathrm{NS}}}{10^{-8} \mathrm{M}_{\odot} \mathrm{yr}^{-1}}\right)\left(\frac{r_{\mathrm{NS}}}{10^{6} \mathrm{~cm}}\right)^{-1} \mathrm{erg} \mathrm{s}^{-1}$.

Thus, for the accretion rates to agree with observations of the $\mathrm{X}$-ray luminosities, we require an initial disk mass of around $10^{-8} \mathrm{M}_{\odot}$ or smaller. This agrees well with the Be star mass loss rates that are in the range $10^{-8}$ to $10^{-10} \mathrm{M}_{\odot} \mathrm{yr}^{-1}$ (e.g. Snow 1981; Porter \& Rivinius 2003) and the lifetimes of the Be star disks (of a few years).

\section{DISCUSSION}

The simulations presented here model a warped, eccentric, precessing Be star disk. The ejection mechanism for material from the Be star to the disk is poorly understood and thus we have considered only the evolution following disk formation. Thus, the disc is an accretion rather than a decretion disc, but over the timescales we simulate this should not affect our conclusions. The ejection of material from the Be Star surface in a circular orbit at the inner edge of the disc could affect the eccentricity growth and inclination of the disc. However, because eccentricity growth is driven more strongly in the outer parts of the disc, this should not affect the Type II outburst mechanism.

During the Type II outburst in our simulation, the Be star disk becomes significantly depleted. About $40 \%$ of the initial disk mass is accreted on to the Be star. This depletion could explain the cycles observed in the optical light curves of Be/X-ray binaries (e.g. Rajoelimanana et al. 2011). With mass ejection from the Be star included, the disk would build up again and we would expect the Type II outbursts to recur. The timescale of recurrence would depend on the accretion rate on to the Be star disk. Parameters such as the eccentricity, misalignment and binary separation could be varied to model other Be/X-ray binary systems and predict the types of outbursts that may occur.

Observations suggest that the Type II outbursts occur when the disk becomes warped. However we have found that this is not a sufficient condition, it is necessary for the disk to become eccentric. An inclined circular disk, which can expand freely as the tidal torques are smaller, becomes eccentric before it becomes large enough for a NS encounter. If there are no outbursts observed in a Be binary system, the binary 

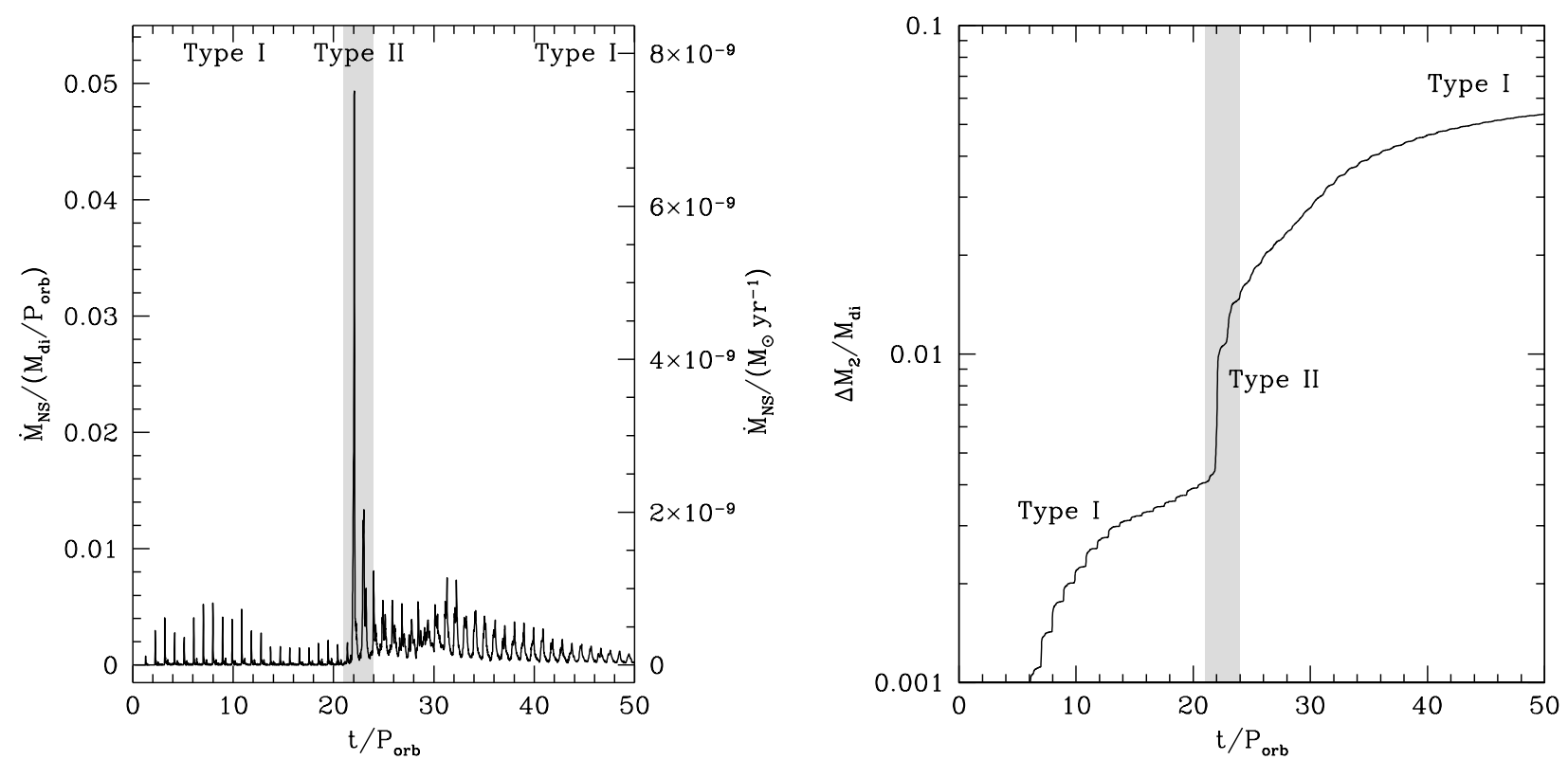

FIG. 4.- Left: The mass transfer rate on to the neutron star (at the accretion radius of $1 \mathrm{R}_{\odot}$ ). The right hand axis shows the equivalent accretion rate in a system with an initial Be star disk mass of $M_{\mathrm{di}}=10^{-8} \mathrm{M}_{\odot}$. Initially the system displays periodic Type I accretion outbursts. From about a time of about 21 to $24 P_{\text {orb }}$, the system undergoes a Type II outburst, where the average accretion rate on to the neutron star is significantly higher. Right: The corresponding total mass accreted on to the neutron star. In each plot the shaded region show the approximate time of the Type II outburst.

must have small eccentricity and misalignment. Conversely, if Type II outbursts are observed, then the system must be highly misaligned. We find that eccentricity may also be driven in counter rotating discs. The frequency of outbursts in $\mathrm{Be} / \mathrm{X}$ ray binary systems will be investigated in future with a disc model including mass injection into the disc from the Be star in order to determine critical inclination angles required for outbursts. The tidal torque aligns the disk with the binary orbital plane on a timescale of years (Martin et al. 2011). In a long term disk simulation this alignment may also play a role in ending the outburst cycle.

\section{CONCLUSIONS}

We have performed 3D SPH simulations of a moderately eccentric and highly misaligned $\mathrm{Be} / \mathrm{X}$-ray binary. We find that for a binary with orbital period of $24 \mathrm{~d}$ and eccentricity 0.34 that Type I outbursts occur for all misalignments, including the aligned case. If the disk is sufficiently misaligned, $i \gtrsim 60^{\circ}$, the disk is much larger because the tidal torque is weaker. This allows strong eccentricity growth in the outer parts of the disk from the gravitational forcing of the eccentric companion. Thus, the disk becomes eccentric on a timescale of around ten binary orbital periods. We suggest that the eccentric disk causes a Type II X-ray outburst as a large amount of material is transferred to the neutron star.

\section{ACKNOWLEDGMENTS}

RGM's support was provided under contract with the California Institute of Technology (Caltech) funded by NASA through the Sagan Fellowship Program. Support for CJN was provided by NASA through the Einstein Fellowship Program, grant PF2-130098. PJA acknowledges support from NASA's ATP program under awards NNX11AE12G and NNX14AB42G. DJP is supported by Future Fellowship FT130100034 from the Australian Research Council. We acknowledge the use of SPLASH (Price 2007) for the rendering of the figures. This work utilised the Janus supercomputer, which is supported by the National Science Foundation (award number CNS-0821794), the University of Colorado Boulder, the University of Colorado Denver, and the National Center for Atmospheric Research. The Janus supercomputer is operated by the University of Colorado Boulder.

\section{REFERENCES}

Artymowicz, P. \& Lubow, S. H. 1994, ApJ, 421, 651

Bjorkman, K. S., Miroshnichenko, A. S., McDavid, D., \& Pogrosheva, T. M. 2002, ApJ, 573, 812

Carciofi, A. C. 2011, in IAU Symposium, Vol. 272, IAU Symposium, ed.

C. Neiner, G. Wade, G. Meynet, \& G. Peters, 325-336

Goldreich, P. \& Tremaine, S. 1980, ApJ, 241, 425

Goodchild, S. \& Ogilvie, G. 2006, MNRAS, 368, 1123

Hanuschik, R. W. 1996, A\&A, 308, 170

Kato, S. 2014, ArXiv e-prints

King, A. R., Pringle, J. E., \& Livio, M. 2007, MNRAS, 376, 1740

Kretschmar, P., Nespoli, E., Reig, P., \& Anders, F. 2013, ArXiv e-prints

Lee, U., Osaki, Y., \& Saio, H. 1991, MNRAS, 250, 432

Lodato, G. \& Price, D. J. 2010, MNRAS, 405, 1212

Lubow, S. H. 1991a, ApJ, 381, 259

-. 1991b, ApJ, 381, 268

-. 1992, ApJ, 401, 317
-. 1994, ApJ, 432, 224

Martin, R. G., Pringle, J. E., Tout, C. A., \& Lubow, S. H. 2011, MNRAS, 416, 2827

Martin, R. G., Tout, C. A., \& Pringle, J. E. 2009, MNRAS, 397, 1563

-. 2010, MNRAS, 401, 1514

Moritani, Y., Nogami, D., Okazaki, A. T., Imada, A., Kambe, E., Honda, S., Hashimoto, O., \& Ichikawa, K. 2011, PASJ, 63, L25

Moritani, Y., Nogami, D., Okazaki, A. T., Imada, A., Kambe, E., Honda, S., Hashimoto, O., Mizoguchi, S., Kanda, Y., Sadakane, K., \& Ichikawa, K. 2013, PASJ, 65, 83

Munar-Adrover, P., Paredes, J. M., Ribó, M., Iwasawa, K., Zabalza, V., \& Casares, J. 2014, ArXiv e-prints

Negueruela, I., Okazaki, A. T., Fabregat, J., Coe, M. J., Munari, U., \& Tomov, T. 2001, A\&A, 369, 117

Negueruela, I., Reig, P., Coe, M. J., \& Fabregat, J. 1998, A\&A, 336, 251

Nixon, C., King, A., \& Price, D. 2013, MNRAS, 434, 1946 
Nixon, C. \& Salvesen, G. 2014, MNRAS, 437, 3994

Nixon, C. J. 2012, MNRAS, 423, 2597

Okazaki, A. T. 2006, Boletin de la Asociacion Argentina de Astronomia La Plata Argentina, 49, 321

Okazaki, A. T. 2007, in Astronomical Society of the Pacific Conference Series, Vol. 367, Massive Stars in Interactive Binaries, ed. N. St.-Louis \& A. F. J. Moffat, 485

Okazaki, A. T., Bate, M. R., Ogilvie, G. I., \& Pringle, J. E. 2002, MNRAS, 337,967

Okazaki, A. T. \& Hayasaki, K. 2007, in Astronomical Society of the Pacific Conference Series, Vol. 361, Active OB-Stars: Laboratories for Stellare and Circumstellar Physics, ed. A. T. Okazaki, S. P. Owocki, \& S. Stefl, 395

Okazaki, A. T., Hayasaki, K., \& Moritani, Y. 2013, PASJ, 65, 41

Okazaki, A. T. \& Negueruela, I. 2001, A\&A, 377, 161

Paczynski, B. 1977, ApJ, 216, 822

Porter, J. M. 1996, MNRAS, 280, L31

Porter, J. M. \& Rivinius, T. 2003, PASP, 115, 1153

Price, D. J. 2007, Pasa, 24, 159

Price, D. J. \& Federrath, C. 2010, MNRAS, 406, 1659
Pringle, J. E. 1981, ARA\&A, 19, 137

-. 1991, MNRAS, 248, 754

Rajoelimanana, A. F., Charles, P. A., \& Udalski, A. 2011, MNRAS, 413, 1600

Reig, P. 2011, Ap\&SS, 332, 1

Reig, P., Larionov, V., Negueruela, I., Arkharov, A. A., \& Kudryavtseva, N. A. 2007, A\&A, 462, 1081

Rivinius, T., Baade, D., Stefl, S., Townsend, R. H. D., Stahl, O., Wolf, B., \& Kaufer, A. 2001, A\&A, 369, 1058

Shakura, N. I. \& Sunyaev, R. A. 1973, A\&A, 24, 337

Shi, J.-M., Krolik, J. H., Lubow, S. H., \& Hawley, J. F. 2012, ApJ, 749, 118 Slettebak, A. 1982, ApJS, 50, 55

Smith, M. A., Hubeny, I., Lanz, T., \& Meylan, T. 1994, ApJ, 432, 392

Snow, Jr., T. P. 1981, ApJ, 251, 139

Stella, L., White, N. E., \& Rosner, R. 1986, ApJ, 308, 669

Underhill, A. B. 1987, in IAU Colloq. 92: Physics of Be Stars, ed.

A. Slettebak \& T. P. Snow, 411-425 